\title{
Synovial Concentration of Trimethoprim-Sulphadiazine Following Regional Limb Perfusion in Standing Horses
}

\author{
Kajsa Gustafsson ${ }^{1}$, Amos J. Tatz ${ }^{1}$, Roee Dahan ${ }^{1}$, Wiessam Abu Ahmad ${ }^{2}$, Malka Britzi ${ }^{3}$, Gila A. Sutton ${ }^{1}$ \\ and Gal Kelmer ${ }^{1, *}$ \\ 1 Department of Large Animal Medicine and Surgery, Koret School of Veterinary Medicine, The Robert H. \\ Smith Faculty of Agriculture, Food and Environment, The Hebrew University of Jerusalem, \\ Rehovot 76100, Israel; kajsagustafsson1@gmail.com (K.G.); tatz@agri.huji.ac.il (A.J.T.); \\ roee.dahan@mail.huji.ac.il (R.D.); gila.sutton@mail.huji.ac.il (G.A.S.) \\ 2 Faculty of Medicine, Hebrew University-Hadassah Braun School of Public Health and Community Medicine, \\ Jerusalem 9112102, Israel; wiessam@gmail.com \\ 3 National Residue Control Laboratory, Kimron Veterinary Institute, Bet Dagan 50250, Israel; \\ malkab@moag.gov.il \\ * Correspondence: gal.kelmer@mail.huji.ac.il
}

check for updates

Citation: Gustafsson, K.; Tatz, A.J.; Dahan, R.; Abu Ahmad, W.; Britzi, M.; Sutton, G.A.; Kelmer, G. Synovial Concentration of

Trimethoprim-Sulphadiazine Following Regional Limb Perfusion in Standing Horses. Animals 2021, 11, 2085. https://doi.org/10.3390/ ani11072085

Academic Editor: Kevin K. Haussler

Received: 25 May 2021

Accepted: 3 July 2021

Published: 13 July 2021

Publisher's Note: MDPI stays neutral with regard to jurisdictional claims in published maps and institutional affiliations.

Copyright: (c) 2021 by the authors. Licensee MDPI, Basel, Switzerland. This article is an open access article distributed under the terms and conditions of the Creative Commons Attribution (CC BY) license (https:/ / creativecommons.org/licenses/by/ $4.0 /)$.
Simple Summary: Trimethoprim-sulphadiazine is a first line antimicrobial drug recommended for use in equine orthopedic infections such as deep wounds, ulcerative lymphangitis and septic arthritis. The pharmacokinetics of trimethoprim-sulphadiazine, when delivered through intravenous regional limb perfusion, has not been previously described. This study aimed at describing the pharmacokinetics and safety of the administration of trimethoprim-sulphadiazine through a single cephalic vein injection. Several horses in the study suffered from severe vasculitis, and the resulting synovial fluid concentration of trimethoprim-sulphadiazine over time was low. In light of these findings, the administration of trimethoprim-sulphadiazine to horses using regional limb perfusion via cephalic appears unwarranted.

Abstract: The aim of this study was to investigate the safety and pharmacokinetics of trimethoprimsulphadiazine administered via intravenous regional limb perfusion (IVRLP) into the cephalic vein. According to the hypothesis, the drug could be administered without adverse effects and the synovial concentrations would remain above the minimum inhibitory concentration (MIC) for trimethoprimsulphadiazine $(0.5$ and $9.5 \mu \mathrm{g} / \mathrm{mL})$ for $24 \mathrm{~h}$. Ten $(n=10)$ horses underwent cephalic vein IVRLP with an Esmarch tourniquet applied for $30 \mathrm{~min}$. Four grams (4 g) of trimethoprim-sulphadiazine (TMP-SDZ) were diluted at $0.9 \% \mathrm{NaCl}$ for a total volume of $100 \mathrm{~mL}$. Synovial fluid and blood samples were obtained immediately before IVRLP and at $0.25,0.5,2,6,12$ and $24 \mathrm{~h}$ after the initiation of IVRLP. Trimethoprim and sulphadiazine concentrations were determined using a method based on liquid chromatography/tandem mass spectrometry. The $\mathrm{C}_{\max }$ (peak drug concentration) values were $36 \pm 31.1$ and $275.3 \pm 214.4 \mu \mathrm{g} / \mathrm{mL}$ (TMP and SDZ). The respective $t_{\max }$ (time to reach $\mathrm{C}_{\max }$ ) values were $20 \pm 7.8$ and $26.4 \pm 7.2 \mathrm{~min}$. The initial synovial fluid concentrations were high but decreased quickly. No horse had synovial concentrations of trimethoprim-sulphadiazine above the MIC at $12 \mathrm{~h}$. Severe vasculitis and pain shortly after IVRLP, lasting up to one week post-injection, occurred in five out of 10 horses. In conclusion, IVRLP with trimethoprim-sulphadiazine cannot be recommended due to the low concentrations of synovial fluid over time and the frequent severe adverse effects causing pain and discomfort in treated horses. Thus, in cases of septic synovitis with bacteria sensitive to trimethoprim-sulphadiazine, other routes of administration should be considered.

Keywords: horse; pharmacokinetics; TMS; regional limb perfusion 


\section{Introduction}

Septic arthritis is a severe and potentially life-threatening infection of synovial structures in horses that has a poor prognosis regarding the return to athletic use or even survival if not treated successfully rapidly [1-4].

Infection of synovial structures of the distal limb can be caused by trauma, iatrogenic intervention (e.g., intra-articular injection or arthroscopic surgery) or via haematogenous spread [5]. The recommended treatment includes an immediate lavage and debridement of the affected synovial structure, preferably under endoscopic guidance, as well as appropriate aggressive antimicrobial therapy [2]. Successful treatment is likely to be dependent on the achievement of high concentrations of antimicrobials within the affected synovial structure. Favorable results are seen when local delivery techniques to the affected synovial structure are used, such as intravenous regional limb perfusion (IVRLP), or antibioticimpregnated polymethyl methacrylate or plaster of Paris beads [6].

The use of intravenous or intraosseous routes for RLP is well described and provides high concentrations of selected antimicrobials to synovial structures, without the adverse effects associated with parental administration, such as colitis or nephrotoxicity [7-10].

Trimethoprim-sulphadiazine combination is a broad-spectrum antimicrobial drug that interrupts the folate metabolism of bacterial cells. The two antimicrobial drugs (trimethoprim and sulphadiazine) exhibit a synergism in inhibiting bacterial DNA synthesis in two stages, reducing the potential for antimicrobial resistance development to a greater extent than when either drug is used alone [11].

Although individually both drugs are bacteriostatic, when combined, trimethoprimsulphadiazine has a broad-spectrum bactericidal antimicrobial activity against many Grampositive and Gram-negative bacteria, although Pseudomonas spp., Mycoplasma spp. and a number of isolates of Klebsiella spp. are resistant [12]. Furthermore, the antimicrobial activity against anaerobic bacteria is poor despite culture and sensitivity tests showing the contrary, most probably due to high levels of folate present in sites of anaerobic infection [12].

With emerging antibiotic resistance in both human and veterinary medicine, an appropriate use of antibiotics and avoidance of the use of some specific antibiotics is advised. Trimethoprim-sulphadiazine is a first-line drug advised for use in orthopedic cases such as deep wounds and septic arthritis, but there are no described methods of local delivery techniques [13]. Additionally, there have been reports of multiresistant Staphylococcus aureus susceptible to trimethoprim-sulphadiazine. In these cases, specifically aimed antimicrobial treatment can be lifesaving [14].

The pharmacokinetics of trimethoprim-sulphadiazine administered by parenteral route have been well described in plasma, urine, synovial fluid, peritoneal fluid, endometrium and pulmonary epithelial lining in horses [15-18]. The established Minimal Inhibitory Concentration (MIC) of trimethoprim and sulphadiazine for the most commonly isolated bacteria in orthopedic infections is $0.5 \mu \mathrm{g} / \mathrm{mL}$ and $9.5 \mu \mathrm{g} / \mathrm{mL}$, respectively [19].

The objectives of this study were to evaluate the safety and the pharmacokinetics of trimethoprim-sulphadiazine when administered via IVRLP to horses. Our hypothesis was that the administration would be safe, with no side effects, and that antimicrobial concentrations would remain above the MIC for $24 \mathrm{~h}$, enabling a once daily administration.

\section{Study Design}

\subsection{Animals}

Ten horses were enrolled to the study, two geldings and eight mares. Their ages ranged from two to 18 years (mean 10 years), and their weights ranged from $285 \mathrm{~kg}$ to $560 \mathrm{~kg}$ (mean $400 \mathrm{~kg}$ ). All horses underwent a thorough clinical examination, complete blood count, as well as serum creatinine, urea and electrolyte analyses. No abnormalities were observed. Dynamic orthopaedic examinations were unremarkable, and the anatomy of the front limbs in all horses appeared normal on palpation and static flexion. The horses were housed individually and had free access to water and hay throughout the study. 
Approval for the study was granted by the IACUC (Institutional Animal Care and Use Committee) of the university (MD-16-14812-3).

\subsection{Regional Limb Perfusion and Sampling}

Perfusion was performed in a similar fashion to previous studies from our laboratory [10]. One forelimb limb of each horse was randomly selected for IVRLP by coin toss. The distal portion of the forelimb was desensitized by anaesthetizing the ulnar, median and medial cutaneous antebrachial nerves with $10 \mathrm{~mL}$ of $2 \%$ mepivacaine $\mathrm{HCl}$ (Mepivacaine, Ceva Animal Health, Glenorie, Australia) per site. After that, hair over the cephalic vein at the level of the chestnut was clipped and prepared for aseptic venipuncture. Horses were sedated with detomidine hydrochloride $(0.01 \mathrm{mg} / \mathrm{kg}$, IV; Domosedan, Orion Pharma, Espoo, Finland) and butorphanol tartrate $(0.01 \mathrm{mg} / \mathrm{kg}$, IV; Butomidor, Richter Pharma AG, Wels, Austria). Two rolled gauzes (Sion Medical, Sderot, Israel), each being $10 \mathrm{~cm}$ long, were placed over the vasculature proximal to the intended site of catheterization. An 8-cm wide rubber tourniquet (Degania Medical, Degania Bet, Israel) was applied forcefully over the gauze rolls and around the limb to occlude blood flow.

A 23-gauge, 2-cm long butterfly catheter (Scalp Vein Set, Zhejiang Kindly Medical Devices Co Ltd., Binhai, China) was inserted in an aseptic manner into the cephalic vein about $5 \mathrm{~cm}$ distal to the tourniquet and aimed distally. The catheter was adhered to the skin with cyanoacrylate glue. A perfusate composed of $16 \mathrm{~mL}$ of trimethoprim-sulphadiazine (Trimethoprim (4\%) Sulphadiazine (20\%), Norbrook Laboratories, Northern Ireland) in $84 \mathrm{~mL}$ of sterile isotonic saline solution (Sodium Chloride, Braun, Melsungen, Germany), giving a total volume of $100 \mathrm{~mL}$, was infused into the cephalic vein over a period of $2 \mathrm{~min}$. The tourniquet was removed $30 \mathrm{~min}$ after the perfusate was injected. Horses were observed closely for abnormalities associated with the injection, such as inadvertent perivascular administration, swelling or signs of pain. The movements of the horse's legs, other than weight shifting, were recorded during the procedure.

\subsection{Synovial Fluid Sampling}

Sampling was performed similarly to previous studies from our laboratory [8]. After preparing the skin of the lateral aspect of the joint for aseptic arthrocentesis, synovial fluid $(1 \mathrm{~mL})$ was collected from the lateral aspect of the metacarpophalangeal joint (MCPJ), with the limb in light flexion. A 21-gauge, 3.81-cm needle was inserted into a slight depression between the proximal phalanx, the proximal sesamoid bone and the third metacarpal bone, through the collateral sesamoidean ligament, until the joint was entered. Synovial fluid $(1 \mathrm{~mL})$ was aspirated into a 3-mL syringe. Synovial fluid was collected after the tourniquet was applied but before the perfusate was injected $(\mathrm{T}=0)$ and at $0.25 \mathrm{~h}$ and $0.5 \mathrm{~h}$ after injection. The tourniquet was removed after the collection of the 30-min sample. Synovial fluid was collected from the same joint at 2, 6, 12 and $24 \mathrm{~h}$ post perfusion. One-gram amikacin sulphate (Amikacin, Anfarm Hellas, Attika, Greece) was injected into the MCPJ following the final synovial fluid sample collection because of the perceived risk of synovial infection caused by repeated arthrocentesis of the joint. The horses were sedated with xylazine $\mathrm{HCl}$ (Hydrogen Chloride) $(0.5 \mathrm{mg} / \mathrm{kg}$, IV; AnaSed, Akorn Animal Health, Vernon Hills, IL, USA) as needed, to prevent discomfort and movement during arthrocentesis. Using a 21-gauge, 3.81-cm needle, blood $(2 \mathrm{~mL})$ was collected aseptically from the jugular vein at the same time points at which the synovial fluid was harvested.

All samples of synovial fluid and blood were placed into a plastic sample tube containing no anticoagulants. After coagulation, the samples were centrifuged for $5 \mathrm{~min}$ at $1000 \mathrm{~g}$, and the supernatant was collected and frozen at $-80^{\circ} \mathrm{C}$. The limb was maintained in a bandage between each collection of synovial fluid and for two days after the final arthrocentesis. All horses received phenylbutazone $(4.4 \mathrm{mg} / \mathrm{kg}$, IV) after the last sampling and for the next two days $(2.2 \mathrm{mg} / \mathrm{kg}$, PO, q12 h). Horses were observed once daily for two weeks for lameness and swelling at the injection sites associated with venipuncture, IVRLP or arthrocentesis. 


\subsection{Determination of Antimicrobial Concentration}

Samples of serum and synovial fluid $(20 \mu \mathrm{L})$ were vortex-mixed with $0.5 \mathrm{~mL}$ bidistilled water (Type 1, Micropure water purification system, Thermo Scientific, Asheville, NC, USA) and $0.5 \mathrm{~mL}$ acetonitrile (J.T. Baker, Deventeer, The Netherlands). After $5 \mathrm{~min}$ of centrifugation at $14,000 \mathrm{rpm}$, at $4{ }^{\circ} \mathrm{C}$, an aliquot of supernatant was used for LC/MS/MS analysis. Calibration curves of suphadiazine (A2S, Saint Jean d'Illac, France) and trimethoprim (Dr. Ehrenstorfer, Germany) were prepared in serum and synovial fluid free from the presence of these compounds, within a concentration range of $0.25-50 \mu \mathrm{g} / \mathrm{mL}$ in synovial fluid and $0.1-5 \mu \mathrm{g} / \mathrm{mL}$ in serum. Samples exceeding the highest level of calibration were diluted and reanalyzed. Accuracy and precision were determined on quality control (QC) samples fortified with $0.25,5$ and $50 \mu \mathrm{g} / \mathrm{mL}$ in synovial fluid and $0.1,1$ and $5 \mu \mathrm{g} / \mathrm{mL}$ in serum. These values represent low-level (LL), medium-level (ML) and high-level (HL) calibration curves.

Sulphadiazine and trimethoprim were detected and quantified by liquid chromatography tandem mass spectrometry (LC/MS/MS). The system included an Agilent 1200 (Agilent Technologies, Waldbronn, Germany) liquid chromatography system (with a degasser, binary pump, temperature-controlled column compartment and autosampler) connected to an Applied Biosystems API 4000 mass spectrometer (Applied Biosystems, Toronto, ON, Canada).

Compounds were separated on a Zorbax, Eclipse Plus C18 column $(1.8 \mu \mathrm{m}, 4.6 \times 50 \mathrm{~mm}$, Agilent, Santa Clara, CA, USA). The mobile phase consisted of $0.1 \%$ formic acid (Sigma, St. Louis, MO, USA) and acetonitrile. The precursor ions were 251 and $291 \mathrm{~m} / \mathrm{z}$ and the quantifier product ions were 156 and $230 \mathrm{~m} / \mathrm{z}$ for sulphadiazine and trimethoprim, respectively.

\subsection{Statistical Methods}

WinPepi; Pairsetc. (Version 3.59); Power calculation P3 was used to calculate the power of the study. The data were assumed not to have a normal distribution based on the histogram and the small sample size. The levels of each antibiotic drug at the six different time points at each of the two locations were compared individually by the nonparametric Friedman Test for a repeated-measures 2-way Analysis of Variance (ANOVA) by ranks. $p$-values for the pair-wise comparisons were adjusted for multiple comparisons by the Bonferroni correction. Statistical significance was set at $p<0.05$. Statistical analysis was carried out in IBM SPSS Version 25.

\section{Results}

During the course of the experimental protocol, five horses developed severe phlebitis of the cephalic vein, with associated cellulitis, which manifested in severe swelling, heat, pain upon palpation of the distal limb and lameness at walking. In one horse, pain and swelling of the distal limb made it inappropriate to perform any further arthrocentesis from $6 \mathrm{~h}$ post-infusion. Nevertheless, data obtained from this horse was included in the study. For the horses with phlebitis, local cryotherapy with ice bandages was applied for the first $24 \mathrm{~h}$, and phenylbutazone $(2.2 \mathrm{mg} / \mathrm{kg}, \mathrm{PO}, \mathrm{q} 12 \mathrm{~h})$ administration was continued until the acute phlebitis resolved for 5-7 days, in addition to the standard two days. In three horses the lameness resolved within 1-2 days, and two horses showed lameness and reluctance to walk that lasted 3-5 days.

The power of the study was $72 \%$ for the sulphadiazine and $70 \%$ for the trimethoprim. Median (lower quartile; upper quartile) trimethoprim and sulphadiazine synovial $\mathrm{C}_{\max }$ values were $19.2(3.46 ; 50.6)$ and $175(21.85 ; 394) \mu \mathrm{g} / \mathrm{mL}$. The respective $t_{\max }$ values were $15(15 ; 30)$ and $15(15 ; 15)$ minutes. The respective median serum $C_{\max }$ values were 3.5 and $65.6 \mu \mathrm{g} / \mathrm{mL}$, and the serum mean $\mathrm{t}_{\max }$ values were $42 \pm 43.8$ and $114 \pm 109.8 \mathrm{~min}$.

The concentrations of trimethoprim and sulphadiazine in synovial fluid were significantly different over time, $p=0.031$ and $p=0.021$ respectively. The concentrations of trimethoprim and sulphadiazine in serum were not significantly different over time, $p=0.14$ and $p=0.05$ respectively. 
Trimethoprim synovial fluid concentrations were initially high but decreased quickly, and only the concentrations at time points 0.25 and $0.5 \mathrm{~h}$ were statistically different from the synovial fluid concentrations at time point T0 $(p<0.01)$. Sulphadiazine synovial fluid concentrations remained elevated for longer, but nevertheless the synovial fluid concentrations at time points $0.25,0.5(p<0.001)$ and $2 \mathrm{~h}(p<0.01)$ were the only time points statistically different from the synovial fluid concentrations at time point $\mathrm{T} 0$. The trimethoprim and sulphadiazine synovial fluid concentration at $0.25 \mathrm{~h}$ was not statistically different from the synovial fluid concentration at time point $0.5 \mathrm{~h}(p=1.00)$.

Since the data were not normally distributed, the synovial concentrations at each time point are described in medians with quartiles (Figures 1 and 2).

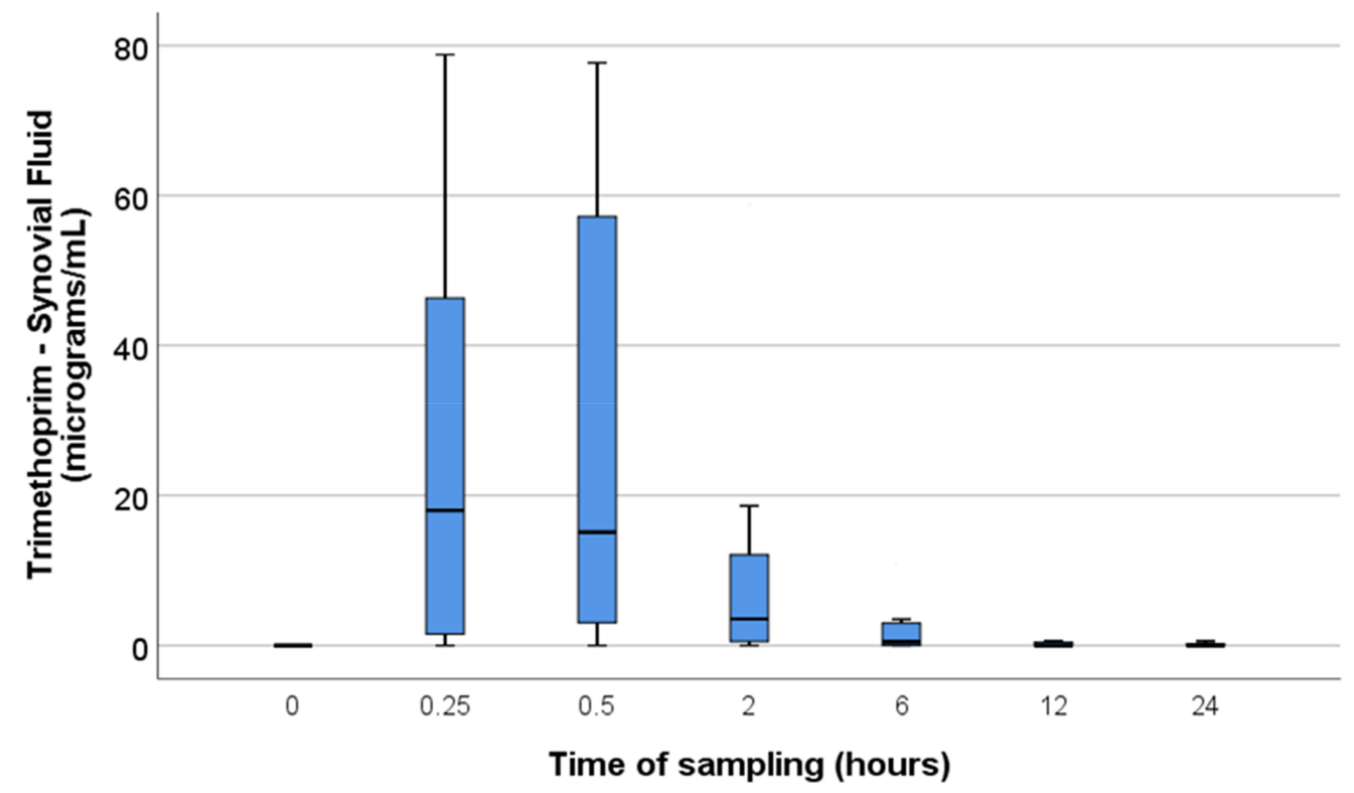

Figure 1. Trimethoprim synovial fluid concentrations at each time point. Median values and quartiles indicated by boxplots with maximum and minimum values indicated by whiskers. Synovial fluid concentrations were only significantly different from the concentrations at $\mathrm{T} 0$ at time points 0.25 and $0.5 \mathrm{~h}$, although the median synovial fluid concentration remained above the MIC $(0.5 \mu \mathrm{g} / \mathrm{mL})$ until the 6-h time point.

The intraday accuracy of the synovial fluid QC samples was $91 \%, 99 \%$ and $94 \%$ for LL, ML and HL of sulphadiazine and 109\%, 104\% and 105\% for trimethoprim. The precision (coefficient of variation) was $15 \%, 8.9 \%$ and $9.5 \%$ for LL, ML and HL of sulphadiazine and $2.1 \%, 5.1 \%$ and $5.4 \%$ for trimethoprim.

The interday accuracy of the serum QC samples was 107\%, 97\% and $94 \%$ for LL, ML and HL of sulphadiazine and $110 \%, 102 \%$ and $103 \%$ for trimethoprim. The precision (coefficient of variation) was $14 \%, 13.4 \%$ and $12.3 \%$ for LL, ML and HL of sulphadiazine and $14.8 \%, 7.1 \%$ and $4.4 \%$ for trimethoprim. 


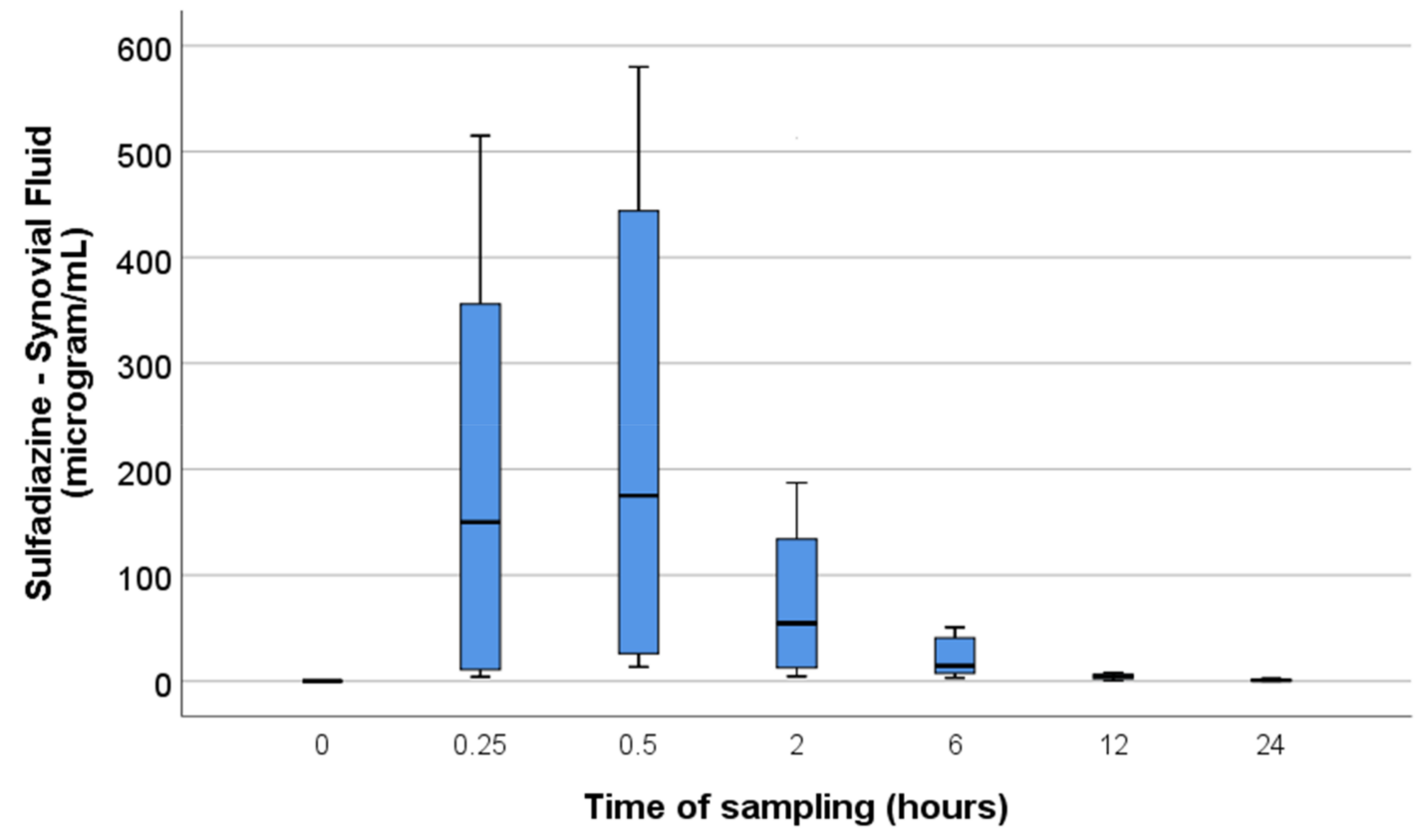

Figure 2. Sulphadiazine synovial fluid concentrations at each time point. Median values and quartiles indicated by boxplots with maximum and minimum values indicated by whiskers. The synovial fluid concentrations were only significantly different from the concentrations at $\mathrm{T} 0$ at time points $0.25,0.5$ and $2 \mathrm{~h}$, although the median synovial fluid concentration remained above the MIC $(9.5 \mu \mathrm{g} / \mathrm{mL})$ until the 6-h time point.

\section{Discussion}

This study aimed at investigating if trimethoprim-sulphadiazine administration to horses by IVRLP was safe and could maintain a synovial fluid concentration above MIC for $24 \mathrm{~h}$.

The results revealed that trimethoprim-sulphadiazine administrated by IVRLP created severe and longstanding, apparently chemically induced, phlebitis in a high percentage of horses. The conclusion was that it cannot be administered safely to horses, at least with this formulation. In addition, the synovial fluid concentration of trimethoprim-sulphadiazine did not remain above MIC for the most common equine pathogens for $24 \mathrm{~h}$, and both hypotheses have to therefore be rejected.

The horses that suffered from phlebitis were diagnosed with chemically induced phlebitis as it occurred in association with the intravenous infusion [20]. Chemically induced phlebitis is usually caused by injection of substances with high or low $\mathrm{pH}(<5$ or $>10)$ or substances with high osmolarity [21]. The trimethoprim-sulphadiazine formulation used in this study had a $\mathrm{pH}$ of 10 , which could explain the reaction. Injection of substances with a substantially different $\mathrm{pH}$ to blood (7.35-7.45) causes inflammatory cell infiltration, edema, thrombosis and cell death via endothelial damage [21]. We could not find reports on vascular reaction to either the active ingredients (Trimethoprim and Sulphadiazine) or the main excipients (Chlorocresol and Sodium Formaldehyde Sulphoxylate Dihydrate). Clinical signs of phlebitis include tenderness, erythema, swelling and a palpable venous cord [22], which were all observed in the affected horses in this study. The decreased endothelial anticoagulant activity of the vessels exposed to irritating substances leads to thrombosis and loss of patency of the vessel. In these cases, thrombophlebitis was diagnosed [21]. Although clinically evident in the present study, the diagnosis of thrombophlebitis was not confirmed by further diagnostic tests such as ultrasonography.

The risk of chemically induced phlebitis can be decreased by diluting the injected substance. However, in this study, $16 \mathrm{~mL}$ of trimethoprim-sulphadiazine was diluted in $84 \mathrm{~mL}$ of saline for a total volume of $100 \mathrm{~mL}$. Intravenous administration of trimethoprimsulphadiazine to humans is prepared by diluting $5 \mathrm{~mL}$ in $75-125 \mathrm{~mL}$ of $5 \%$ dextrose, a 
higher dilution than was used in this study. Additionally, the 5\% dextrose in sterile water is used as a diluent in humans because it mimics the osmolarity of blood and is thought to decrease the risk of a vascular reaction [20]. The phlebitis observed this study could potentially have been avoided by a higher dilution of the antimicrobial solution. However, a volume higher than $100 \mathrm{~mL}$ has not been investigated in IVRLP of the horse distal limb. Additionally, the administration time of trimethoprim-sulphadiazine in humans is recommended to be 60-90 $\mathrm{min}$ in order to obtain a satisfactory hemodilution during the infusion. In IVRLP, although the trimethoprim-sulphadiazine was diluted, the rapid injection time that was necessary, combined with the presence of a tourniquet when administering a drug through IVRLP, might not have provided sufficient hemodilution, resulting in the exposure of the tunica intima of the vein to high concentrations of the drug.

The dosage chosen in this study was based on the proposed dosage of $1 / 3$ of the systemic dose when administering concentration-dependent antimicrobials through IVRLP [3]. The concentration-dependent activity of trimethoprim-sulphadiazine is debatable and although some concentration-dependent mode of action is evident, some authors also suggest a time-dependent activity of trimethoprim-sulphadiazine [23]. If a time-dependent mode of action were assumed, the dose would have to be increased to a full systemic dose, most likely resulting in even more detrimental effects on the vascular endothelium.

The low synovial fluid concentrations over time are particularly ineffective when using trimethoprim-sulphadiazine. Although the combination of trimethoprim-sulphadiazine is synergistic and can exert a bactericidal effect, both antimicrobial drugs are mainly bacteriostatic, and it is thus important to keep blood concentrations above MIC for as long a period of time as possible [15].

The volume of injection used in this study $(100 \mathrm{~mL})$ was based on previous studies concluding that a higher volume of infusion led to higher synovial fluid concentrations of the infused antimicrobial [24,25].

The synovial fluid concentration of trimethoprim-sulphadiazine was highly variable among the horses. The reason for the large variation could be related to several factors including blood contamination of synovial samples [26], transport of the perfusion to the systemic circulation through intraosseous vessels [27], or failure of the tourniquet caused by a faulty application or movement of the horse [28-30].

The use of Esmarch rubber tourniquets in this study was elected in order to mimic the most probable clinical setting. Although the use of pneumatic tourniquets would provide a more consistent protocol, the Esmarch rubber tourniquet has proved to be equally useful in this type of experimental protocol [31].

The fetlock was chosen as the sample site because in most other RLP PK studies that we performed [8-10,24,29] and in many other RLP PK studies [3,31], the fetlock was sampled. Conforming to a uniform and previously established study design is advantageous.

This study has several limitations including the small study group with highly variable synovial fluid trimethoprim-sulphadiazine concentrations. The sample size was based on previous studies where the sample size was large enough to find significant differences $[24,25,28,29]$. The same volume of antimicrobial was infused despite significant differences in body weight, and the antimicrobial concentrations were only measured in synovial fluid from the metacarpophalangeal joint. Thus, the antimicrobial concentration in other joints, skin, subcutaneous tissue and bone is unknown.

\section{Conclusions}

Based on the induction of severe and longstanding chemically induced phlebitis and a low synovial fluid concentration over time, trimethoprim-sulphadiazine could not be recommended for administration to horses via IVRLP.

Author Contributions: Conceptualization, G.K., K.G. and A.J.T.; methodology, G.K.; formal analysis, M.B.; investigation, G.K., A.J.T., R.D., K.G.; resources, G.K. and M.B.; data curation, W.A.A. and G.A.S.; writing—original draft preparation, K.G.; writing—review and editing, K.G., G.A.S. and G.K.; 
supervision, R.D. and A.J.T.; project administration, A.J.T., K.G. and G.K.; funding acquisition, G.K. All authors have read and agreed to the published version of the manuscript.

Funding: The study was funded by an internal hospital grant.

Institutional Review Board Statement: Approval for the study was granted by the IACUC (Institutional Animal Care and Use Committee) of the university (MD-16-14812-3).

Data Availability Statement: All original data is available upon request from the authors.

Conflicts of Interest: The authors declare no conflict of interest related to this report.

\section{References}

1. Kelmer, G. Regional Limb Perfusion in Horses. Vet. Rec. 2016, 178, 581-584. [CrossRef]

2. Kelmer, G.; Tatz, A.; Bdolah-Abram, T. Indwelling Cephalic or Saphenous Vein Catheter Use for Regional Limb Perfusion in 44 Horses with Synovial Injury Involving the Distal Aspect of the Limb. Vet. Surg. 2012, 41, 938-943. [CrossRef]

3. Rubio-Martínez, L.M.; Cruz, A.M. Antimicrobial Regional Limb Perfusion in Horses. J. Am. Vet. Med. Assoc. 2006, $228,706-712$. [CrossRef] [PubMed]

4. Rubio-Martínez, L.M.; Elmas, C.R.; Black, B.; Monteith, G. Clinical Use of Antimicrobial Regional Limb Perfusion in Horses: 174 Cases (1999-2009). J. Am. Vet. Med. Assoc. 2012, 241, 1650-1658. [CrossRef]

5. Cousty, M.; Stack, J.D.; Tricaud, C.; David, F. Effect of Arthroscopic Lavage and Repeated Intra-Articular Administrations of Antibiotic in Adult Horses and Foals with Septic Arthritis. Vet. Surg. 2017, 46, 1008-1016. [CrossRef] [PubMed]

6. Cruz, A.M.; Rubio-Martinez, L.; Dowling, T. New Antimicrobials, Systemic Distribution, and Local Methods of Antimicrobial Delivery in Horses. Vet. Clin. N. Am. Equine Pract. 2006, 22, 297-322. [CrossRef]

7. Scheuch, B.C.; Van Hoogmoed, L.M.; Wilson, W.D.; Snyder, J.R.; MacDonald, M.H.; Watson, Z.E.; Steffey, E.P. Comparison of Intraosseous or Intravenous Infusion for Delivery of Amikacin Sulfate to the Tibiotarsal Joint of Horses. Am. J. Vet. Res. 2002, 63, 374-380. [CrossRef] [PubMed]

8. Kelmer, G.; Bell, G.C.; Martin-Jimenez, T.; Saxton, A.M.; Catasus, C.; Elliot, S.B.; Meibohm, B. Evaluation of Regional Limb Perfusion with Amikacin Using the Saphenous, Cephalic, and Palmar Digital Veins in Standing Horses. J. Vet. Pharm. Therap. 2013, 36, 236-240. [CrossRef]

9. Kelmer, G.; Tatz, A.J.; Famini, S.; Bdolah-Abram, T.; Soback, S.; Britzi, M. Evaluation of Regional Limb Perfusion with Chloramphenicol Using the Saphenous or Cephalic Vein in Standing Horses. J. Vet. Pharm. Therap. 2015, 38, 35-40. [CrossRef]

10. Kelmer, G.; Tatz, A.J.; Kdoshim, E.; Britzi, M.; Segev, G. Evaluation of the Pharmacokinetics of Imipenem Following Regional Limb Perfusion Using the Saphenous and the Cephalic Veins in Standing Horses. Res. Vet. Sci. 2017, 114, 64-68. [CrossRef]

11. Reinemeyer, C.; Koenig, R.; Hawkins, P.A. A Controlled Safety Study of Elevated Dosages of Trimethoprim Plus Sulfadiazine in Mature Horses. J. Equine Vet. Sci. 2014, 34, 626-631. [CrossRef]

12. Haggett, E.F.; Wilson, W.D. Overview of the Use of Antimicrobials for the Treatment of Bacterial Infections in Horses. Equine Vet. Educ. 2008, 20, 433-448. [CrossRef]

13. Weese, J.S.; Baptiste, K.E.; Baverud, V.; Toutain, P.-L. Guidelines for Antimicrobial Use in Horses. In Guide to Antimicrobial Use in Animals; John Wiley \& Sons, Ltd.: Hoboken, NJ, USA, 2008; pp. 161-182.

14. Steinman, A.; Masarwa, S.; Tirosh-Levy, S.; Gleser, D.; Kelmer, G.; Adler, A.; Carmeli, Y.; Schwaber, M.J. Methicillin-Resistant Staphylococcus Aureus Spa Type T002 Outbreak in Horses and Staff at a Veterinary Teaching Hospital after Its Presumed Introduction by a Veterinarian. J. Clin. Microbiol. 2015, 53, 2827-2831. [CrossRef] [PubMed]

15. Gustafsson, A.; Båverud, V.; Franklin, A.; Gunnarsson, A.; Ogren, G.; Ingvast-Larsson, C. Repeated Administration of Trimethoprim/Sulfadiazine in the Horse-Pharmacokinetics, Plasma Protein Binding and Influence on the Intestinal Microflora. J. Vet. Pharm. Therap. 1999, 22, 20-26. [CrossRef]

16. Bertone, A.L.; Jones, R.L.; McIlwraith, C.W. Serum and Synovial Fluid Steady-State Concentrations of Trimethoprim and Sulfadiazine in Horses with Experimentally Induced Infectious Arthritis. Am. J. Vet. Res. 1988, 49, 1681-1687. [PubMed]

17. Brown, M.P.; Gronwall, R.; Castro, L. Pharmacokinetics and Body Fluid and Endometrial Concentrations of TrimethoprimSulfamethoxazole in Mares. Am. J. Vet. Res. 1988, 49, 918-922.

18. Winther, L.; Guardabassi, L.; Baptiste, K.E.; Friis, C. Antimicrobial Disposition in Pulmonary Epithelial Lining Fluid of Horses. Part I. Sulfadiazine and Trimethoprim. J. Vet. Pharm. Therap. 2011, 34, 277-284. [CrossRef]

19. Sadaka, C.; Kanellos, T.; Guardabassi, L.; Boucher, J.; Watts, J.L. Evaluation of Veterinary-Specific Interpretive Criteria for Susceptibility Testing of Streptococcus Equi Subspecies with Trimethoprim-Sulfamethoxazole and Trimethoprim-Sulfadiazine. J. Clin. Microbiol. 2017, 55, 326-330. [CrossRef]

20. Kokotis, K. Preventing Chemical Phlebitis. Nursing 1998, 28, 41. [CrossRef]

21. Everitt, N.J.; Krupowicz, D.W.; Evans, J.A.; McMahon, M.J. Ultrasonographic Investigation of the Pathogenesis of Infusion Thrombophlebitis. Br. J. Surg. 1997, 84, 642-645.

22. Maki, D.G.; Ringer, M. Risk Factors for Infusion-Related Phlebitis with Small Peripheral Venous Catheters. Ann. Intern. Med. 1991, 114, 845-854. [CrossRef] [PubMed]

23. Brown, G.R. Cotrimoxazole-Optimal Dosing in the Critically Ill. Ann. Intens. Care 2014, 4, 13. [CrossRef] [PubMed] 
24. Oreff, G.L.; Dahan, R.; Tatz, A.J.; Raz, T.; Britzi, M.; Kelmer, G. The Effect of Perfusate Volume on Amikacin Concentration in the Metacarpophalangeal Joint Following Cephalic Regional Limb Perfusion in Standing Horses. Vet. Surg. 2016, 45, 625-630. [CrossRef] [PubMed]

25. Godfrey, J.L.; Hardy, J.; Cohen, N.D. Effects of Regional Limb Perfusion Volume on Concentrations of Amikacin Sulfate in Synovial and Interstitial Fluid Samples from Anesthetized Horses. Am. J. Vet. Res. 2016, 77, 582-588. [CrossRef]

26. Roquet, I.; Hendrick, S.; Carmalt, J.L. The Effect of Blood Contamination on Equine Synovial Fluid Analysis. Vet. Comp. Orthop. Traumatol. 2012, 25, 460-465. [CrossRef]

27. Coleman, M.M.; Peng, P.W.; Regan, J.M.; Chan, V.W.; Hendler, A.L. Quantitative Comparison of Leakage under the Tourniquet in Forearm versus Conventional Intravenous Regional Anesthesia. Anesth. Analg. 1999, 89, 1482-1486.

28. Mahne, A.T.; Rioja, E.; Marais, H.J.; Villarino, N.F.; Rubio-Martinez, L.M. Clinical and Pharmacokinetic Effects of Regional or General Anaesthesia on Intravenous Regional Limb Perfusion with Amikacin in Horses. Equine Vet. J. 2014, 46, 375-379. [CrossRef]

29. Oreff, G.L.; Tatz, A.J.; Dahan, R.; Segev, G.; Haberman, S.; Britzi, M.; Kelmer, G. Pharmacokinetics of Ceftazidime after Regional Limb Perfusion in Standing Horses. Vet. Surg. 2017, 46, 1120-1125. [CrossRef]

30. Beccar-Varela, A.M.; Epstein, K.L.; White, C.L. Effect of Experimentally Induced Synovitis on Amikacin Concentrations after Intravenous Regional Limb Perfusion. Vet. Surg. 2011, 40, 891-897. [CrossRef]

31. Levine, D.G.; Epstein, K.L.; Ahern, B.J.; Richardson, D.W. Efficacy of Three Tourniquet Types for Intravenous Antimicrobial Regional Limb Perfusion in Standing Horses. Vet. Surg. 2010, 39, 1021-1024. [CrossRef] 